\title{
DISPONIBILIDADE DE ALIMENTO COLETADO POR OPERÁRIAS DA ABELHA AFRICANIZADA EM FUNÇÃO DOS FATORES AMBIENTAIS
}

\section{DISPONIBILITY OF COLLECTFOOD FOR AFRICANIZED HONEY BEE IN FUNCTION OF ENVIRONMENTAL FACTORS}

\author{
Adhemar PEGORARO' \\ Anselmo CHAVES NETO²
}

\begin{abstract}
RESUMO
O experimento foi conduzido no Município de Mandirituba, Paraná, em novembro de 1999. Objetivou-se avaliar a freqüência com que as operárias das abelhas africanizadas coletaram alimento nas flores de flor-das-almas (Senecio brasiliensis Less.). Em média, o néctar foi o recurso alimentar que apareceu com maior freqüência das sete às dezoito horas. Observou-se que, durante as horas do dia, existem diferenças estatisticamente significativas na variável concentração de açúcares no néctar. Os dados percentuais das variáveis correspondentes aos recursos alimentares apresentaram as seguintes médias: néctar $30,07 \%$, pólen $21,26 \%$, néctar e pólen $20,19 \%$ e operárias sem alimento $28,68 \%$. A correlação apresentou-se significativa, positiva e moderada com a concentração de açúcares no néctar. A correlação entre a concentração de açúcares no néctar com a temperatura é direta positiva e moderada; com a umidade relativa do ar é moderada e inversa com a concentração de açúcares no néctar.
\end{abstract}

Palavras-chave: abelha africanizada, Senecio brasiliensis e concentração de açúcares no néctar.

\begin{abstract}
The experiment was carried out in Mandirituba, state of Paraná, on November 1999. The objective was to evaluate the frequency which honeybee workers visit Senecio brasiliensis flowers to collect nectar and pollen. Nectar was the food resource that showed the highest frequency from 7 a.m. to 6 p.m. with statistically significant differences observed between the hours of the day. The correlation between the sugar concentration in the nectar with temperature is direct positive and moderate; with the air relative humidity it is moderate and inverse.
\end{abstract}

Key-words: africanized; Senecio brasiliensis and sugar concentration in nectar.

\footnotetext{
${ }^{1}$ Eng. Agrônomo Dr. Professor de Apicultura do Departamento de Zootecnia da Universidade Federal do Paraná, rua Marco Pollo, 717 casa 08 , Curitiba, Paraná CEP 82820260, apegoraro@ufpr.br;

${ }^{2}$ Eng Civ Dr. Professor do Departamento de Estatística-UFPR anselmo@est.ufpr.br - C.P. 19081 Centro Politécnico Curitiba PR.
} 


\section{INTRODUÇÃO}

Segundo Lorenzi (1982), a flor-das-almas (Senecio brasiliensis Less.), pertence á família Asteraceae, é uma planta perene, herbácea, ramificada, medindo de 80 a $160 \mathrm{~cm}$ de altura e se reproduz por semente. Ela ocorre no Sul e Sudeste do Brasil, Sul da Bahia, Sul de Goiás e Sul do Mato Grosso do Sul, sendo considerada planta medicinal, porém tóxica para bovinos, eqüinos e ovinos.

A flor-das-almas é uma planta de interesse apícola classificada com valor oito (WIESE, 1985). Porém, não se conhece o horário no qual as abelhas operárias coletam alimento com maior freqüência nesta planta.

O objetivo deste trabalho foi avaliar o percentual de abelhas que portam cada um dos tipos de alimento (néctar, pólen ou ambos) por horário específico, verificar se existe diferença significativa entre os horários específicos (horas do dia) e estimar a matriz de correlação entre a concentração entre as variáveis de açúcares no néctar da planta, temperatura, umidade relativa do ar e luminosidade.

Segundo Gomes et al. (1998), há vários estudos sobre a proporção e a importância da interação entre $A$. mellifera $L$. com a flora, a fauna e os ecossistemas. Porém, há pouca informação sobre o manejo para melhorar o pasto apícola.

\section{MATERIAL E MÉTODO}

Elegeu-se a flor-das-almas (Senecio brasiliensis Lees.) para este experimento porque é uma espécie nativa que ocorre espontaneamente no estágio inicial da sucessão vegetal (capoeira) principalmente, na Floresta com Araucária e disponibiliza alimento o dia inteiro para as operárias de abelhas africanizadas.

Este estudo foi conduzido em plantas floridas de flor-das-almas, que atraíram operárias campeiras de um apiário com 18 colônias de abelhas africanizadas localizado a $200 \mathrm{~m}$ das oito plantas amostradas. O apiário está localizado em Mandirituba, Paraná, longitude $49 \circ 19$ ' 34" W, latitude 25 19 ' 44" $S$ e altitude em torno de $840 \mathrm{~m}$.

O delineamento experimental aplicado foi o de blocos inteiramente casualizados (BOX et al., 1978). As horas do dia, das sete às dezoito horas, em que as operárias forrageavam constituem os níveis do fator horário (tratamentos). O experimento foi blocado segundo os dias em que o delineamento foi conduzido. A variável concentração de açúcares no néctar das operárias campeiras foi a variável resposta. O modelo para a variável resposta é dado pela seguinte expressão:

$$
Y_{i j}=\mu+\alpha_{i}+\beta_{i}+(\alpha \beta)_{i j}+\varepsilon_{i j k}
$$

onde, $Y_{i j}$ é a resposta do experimento, $\alpha_{i}$ é o efeito do nível i do fator hora, $\beta$. é o efeito do nível j do fator dia (bloco), $(\alpha \beta)_{i j}$ é o efeito da interação entre os fatores (hora e dia) e $\varepsilon_{\mathrm{ijk}}$ é o erro inerente ao experimento.
As amostras de abelhas foram coletadas sobre as flores de flor-das-almas com rede entomológica, durante sete dias em novembro de 1999, de hora em hora, no período das sete às dezoito horas.

Nos períodos das sete às nove horas e das dezessete às dezoito horas ocorreu baixa freqüência de operárias campeiras sobre as flores. Assim, nestes períodos as parcelas foram estabelecidas com número de operárias campeiras que variou de sete a 15, e no intervalo das nove às dezesseis horas, as amostras foram de 15 operárias forrageiras.

Estimou-se, com base nessas amostras, as percentagens médias de operárias campeiras que transportavam néctar, pólen, ambos ou que estavam sobre as flores da espécie em estudo, mas sem alimento disponíveis nas operárias. Para isso, as operárias campeiras foram anestesiadas e avaliouse a concentração de açúcares com refratômetro de campo, conforme propôs Juliano (1972).

Foi construída a matriz de correlação para a variável concentração de açúcar no néctar das flores de flor-das-almas com os fatores ambientais temperatura, luminosidade e umidade relativa do ar.

A temperatura e a umidade relativa do ar foram observadas de hora em hora, durante os sete dias, com higrotermômetro digital de mão, conforme descreveu Nicolson (1994).

A luminosidade foi obtida diretamente com luxímetro a campo e também foi amostrada de hora em hora, conforme Funari et al. (1996) com modificação (amostrada ao nível do solo a plena luz).

A premissa de Gaussianidade na variável resposta, necessária à aplicação da Análise de Variância Clássica que é o método indicado para testar a hipótese nula de médias iguais nos níveis, ou seja, $\mathrm{H}_{0}: \mu_{1}=\mu_{2}=\ldots .==_{k}=\mu$, foi testada pelas técnicas de Kolmogorov-Smirnov e Shapiro-Wilks. Na inexistência dessa condição e quanto não apareceu diferença entre os níveis dos blocos (dias) aplicou-se o teste não paramétrico de Kruskal-Wallis para testar a hipótese nula, agora em termos das medianas $H_{0}: \eta_{1}=\eta_{2}=$ $\ldots . .=\eta_{k}=\eta$. A homogeneidade de variância nos resíduos, que também é uma premissa à aplicação da Análise da Variância Clássica, também foi testada segundo o método de Cochran, pois os experimentos foram todos equilibrados.

\section{RESULTADOS}

Em flores de flor-das-almas, no intervalo das sete às dezoito horas, os percentuais médios para os alimentos foram: néctar $30,07 \%$, pólen $21,26 \%$, néctar e pólen $20,19 \%$. Já o percentual médio de operárias sem alimento foi de $28,68 \%$. Para comparação dos níveis médios de concentração de açúcares no néctar, de início investigou-se a Gaussianidade dos resíduos do modelo, pelo teste de Kolmogorov-Smirnov que forneceu $p=0,449$. A homogeneidade da variância também foi testada resultando em $p=0,244$. Portanto, os resíduos são Gaussianos e a homogeneidade de variância também ocorre. Sendo assim, aplicou-se a 
ANOVA clássica no teste da hipótese nula $\mathrm{H}_{0}: \mathrm{H}_{0}: \mu_{1}=$ $\mu_{7}=\ldots .=\mu_{18}=\mu$, resultando em $p=0,000$. Donde se conclui que existe diferença entre as médias de concentração de açúcares no néctar para as horas do dia. Os resultados da comparação múltipla desses níveis resultaram na Tabela 1.

TABELA 1 - Teste de comparação múltipla.

\begin{tabular}{ccc}
\hline Horas & Médias & Grupos Homogêneos \\
\hline 7 & 17,44 & $\mathrm{x}$ \\
8 & 19,30 & $\mathrm{xx}$ \\
9 & 21,06 & $\mathrm{xx}$ \\
11 & 24,01 & $\mathrm{xx}$ \\
18 & 24,66 & $\mathrm{xx}$ \\
10 & 24,92 & $\mathrm{xxx}$ \\
13 & 26,57 & $\mathrm{xxxx}$ \\
15 & 27,08 & $\mathrm{xxx}$ \\
14 & 27,34 & $\mathrm{xx}$ \\
12 & 27,83 & $\mathrm{x}$ \\
16 & 28,48 & $\mathrm{x}$ \\
\hline
\end{tabular}

Os resultados de análise semelhante para as outras variáveis, ou seja, percentual de abelhas que coletavam e transportavam néctar, pólen e ambos os alimentos, estão resumidos na Tabela 2. Sendo que as hipóteses iniciais são:
$\mathrm{H}_{0}: \mu_{\mathrm{N} 7}=\mu_{\mathrm{N} 8}=\ldots .=\mu_{\mathrm{N} 18}=\mu_{\mathrm{N}}$, onde $\mathrm{N}=$ néctar.

$\mathrm{H}_{0}: \mu_{\mathrm{P} 7}=\mu_{\mathrm{P} 8}=\ldots .=\mu_{\mathrm{P} 18}=\mu_{\mathrm{p}}$, onde $\mathrm{P}=$ pólen. néctar e pólen.
$\mathrm{H}_{0}: \mu_{\mathrm{NP7}}=\mu_{\mathrm{NP} 8}=\ldots .=\mu_{\mathrm{NP} 18}=\mu_{\mathrm{NP}}$, onde NP $=$

TABELA 2 - Valores $p$ dos testes de: Gaussianidade, homogeneidade da variância, F da ANOVA e Kruskal-Wallis.

\begin{tabular}{llll}
\hline Tipo de Alimento & $\begin{array}{l}\text { Teste de Gaussianidade } \\
\text { (Kolmogorov-Smirnov) }\end{array}$ & $\begin{array}{l}\text { Homogeneidade de } \\
\text { Variâncias (Cochran) }\end{array}$ & ANOVA ou Kruskal-Wallis \\
\hline Néctar & $p=0,45$ & $p=0,76$ & $p=0,24$ (ANOVA) \\
Pólen & $p=0,26$ & $p=0,027$ & $\begin{array}{l}p=0,0000 \\
\text { (Kruskal-Wallis) } \\
\text { Néctar/Polen }\end{array}$ \\
& $p=0,38$ & $p=0,25$ & $\begin{array}{l}p=0,0002 \\
\text { (ANOVA) }\end{array}$ \\
\hline
\end{tabular}

No caso do pólen não existiu diferença entre os níveis do fator ou blocos (dias), e como ocorreu heterocedasticidade aplicou-se o teste de Kruskal-Wallis.

A análise dos dados apresentada na Tabela 2 evidencia a existência de diferença estatisticamente significativa entre as horas do dia nas variáveis pólen e néctar/pólen, mas isto não ocorreu na variável néctar. Assim, nas variáveis pólen e néctar/pólen aplicou-se o teste de comparação múltipla do tipo DMS (diferença mínima significativa) para verificar quais os períodos horários são diferentes. Os resultados são apresentados na Tabela 3.

TABELA 3 - Comparação múltipla a 5\% significância p/pólen e nectar por hora, em novembro de 1999, das 7 às 18 horas em Mandirituba, PR.

\begin{tabular}{|c|c|c|c|c|c|}
\hline Horas & Médias & $\mathrm{GHp}$ & Horas & Média & $\mathrm{GH} \mathrm{np}$ \\
\hline 17 & 13,21 & $\mathrm{x}$ & 11 & 13,21 & $\mathrm{x}$ \\
\hline 18 & 13,42 & $x$ & 08 & 13,41 & $x$ \\
\hline 16 & 15,29 & $x x$ & 18 & 15,29 & $\mathrm{x}$ \\
\hline 14 & 15,61 & $x X x$ & 15 & 15,61 & $x$ \\
\hline 15 & 16,39 & $x x x$ & 12 & 16,39 & $x x$ \\
\hline 7 & 17,12 & $x x x$ & 07 & 17,12 & $x x$ \\
\hline 9 & 18,35 & $x x x$ & 17 & 18,35 & $x x x$ \\
\hline 13 & 20,58 & $x x$ & 13 & 20,58 & $x x x$ \\
\hline 10 & 21,09 & $x x$ & 09 & 21,09 & $x x x$ \\
\hline 12 & 24,62 & $x x$ & 16 & 24,62 & $x x$ \\
\hline 8 & 26,65 & $x x$ & 14 & 26,55 & $x x$ \\
\hline 11 & 34,57 & $\mathrm{x}$ & 10 & 34,56 & $\mathrm{x}$ \\
\hline
\end{tabular}

$\mathrm{GH}$ = grupos homogêneos para percentagem de abelhas que coletavam pólen.

$\mathrm{G} \mathrm{Hnp=} \mathrm{grupos} \mathrm{homogêneos} \mathrm{para} \mathrm{a} \mathrm{percentagem} \mathrm{de} \mathrm{abelhas} \mathrm{que} \mathrm{transportavam} \mathrm{néctar} \mathrm{e} \mathrm{pólen.}$ 
PEGORARO A. e CHAVES NETO, A. Disponibilidade de alimento...

$\mathrm{Na}$ Tabela 4 é apresentada a matriz de correlação entre as variáveis: concentração de açúcares no néctar, temperatura, umidade relativa do ar e luminosidade. Nas entradas da tabela estão: a correlação, tamanho da amostra de pares e valor-p.

TABELA 4 - Matriz de correlação entre as variáveis aleatórias, concentração de açúcares no néctar de flor-das-almas, temperatura, umidade relativa do ar e luminosidade.

\begin{tabular}{ccccc}
\hline & \% açúcares & $\begin{array}{c}\text { Temperatura } \\
\text { do ar }\end{array}$ & Umidade do ar & Luminosidade \\
\hline Açúcares\% & & 0,64 & $-0,59$ & 0,13 \\
Temperatura do ar & 0,65 & $(84)$ & $(84)$ & $(84)$ \\
& $(84)$ & 0,00 & 0,00 & 0,21 \\
Umidade do ar & 0,00 & & $-0,53$ & 0,39 \\
& $-0,59$ & $-0,53$ & $(84)$ & $(84)$ \\
Luminosidade & $(84)$ & $(84)$ & 0,00 & 0,00 \\
& 0,00 & 0,00 & & 0,19 \\
& 0,13 & 0,39 & 0,19 & 0,06 \\
& $(84)$ & $(84)$ & $(84)$ & \\
\hline
\end{tabular}

Observa-se que as correlações são quase todas significativas, exceto a correlação entre luminosidade e concentração de açúcares. A correlação entre a concentração de açúcares no néctar e a temperatura apresentou coeficiente de 0,65 sendo significativa, positiva e moderada. Entre a concentração de açúcares no néctar e à umidade relativa do ar a correlação é significativa, negativa e moderada com coeficiente de - 0,59, mostrando de forma clara que aumentando a umidade do ar, diminui a concentração de açúcares.

A luminosidade apresentou coeficiente de correlação com a concentração de açúcares no néctar de $r=0,14$, correlação esta, não significativa.

\section{DISCUSSÃO}

Quanto à percentagem de abelhas transportando os alimentos: néctar, pólen e ambos e, ainda, operárias que não transportavam alimento os dados mostram uma aproximação com os relatados por Funari et al. (1996). É importante destacar que estes autores fizeram uma avaliação em ambiente de clima tropical e as coletas foram realizadas no alvado das colméias.

Os dados deste trabalho mostraram que existe diferença significativa entre as horas do dia quanto a variável concentração de açúcares no néctar das flores do arbusto nativo flor-das-almas, sendo este resultado semelhante ao encontrado no arbusto fruto-de-pombo (Rhamunus sphaerosperma S. W.) Pegoraro (2003).

A concentração média diária de açúcares no néctar está próxima da média observada por Wiese (1985) e Funari et al. (1996), ou seja, é de 24,79 \pm $4,74 \%$.

$\mathrm{Na}$ Tabela 1 observa-se que a menor concentração de açúcares no néctar ocorreu entre sete e oito horas. Isso se deve às condições climáticas adversas, tais como: temperatura baixa e umidade relativa do ar alta. Estes resultados estão em concordância com Funari (1996) e Pegoraro (2003). Assim, como a concentração de açúcares no néctar é baixa, ela exige mais trabalho para as operárias transformarem o néctar em mel (PEGORARO e CARPANEZZI, 1995).

As maiores concentrações de açúcares no néctar ocorreram entre as dezesseis e dezessete horas. Provavelmente, nas últimas horas do dia as flores de flor-das-almas estavam finalizando o ciclo de secreção de néctar e a antese, pois, apesar de apresentar a maior concentração média de açúcares no néctar, a freqüência de operárias campeiras sobre as flores da espécie em estudo foi baixa, possivelmente elas apresentavam volume de néctar baixo e conseqüentemente a recompensa era a menor do dia. Esse comportamento também foi observado por Pegoraro (2003) e está conforme observaram Cane e Schiffhauer (1997). Deste modo conclui-se que as operárias da $A$. mellifera preferem visitar flores nas horas que elas oferecem maior recompensa nutricional. Ás onze horas ocorreu a maior percentagem de operarias que coletavam pólen Tabela 3 e a maior percentagem de operárias que coletavam néctar e pólen ocorreu às dez horas (Tabela 3 ).

A relação entre os fatores ambientais segue o modelo geral, ou seja, quando a temperatura aumenta, a umidade relativa do ar diminui conforme o modelo observado por Nicolson (1998) e Pegoraro (2003).

\section{CONCLUSÕES}

Pode-se concluir que nas condições em que o experimento foi realizado, a concentração de açúcares no néctar apresenta diferenças 
estatisticamente significativas entre as horas do dia, sendo que os maiores valores são observados entre 13 e 17 horas;

Não há diferença estatisticamente significativa entre as horas do dia quanto á quantidade abelhas que coletavam néctar;

O horário de preferência para as abelhas coletar pólen é às 11 horas;

A hora com maior freqüência de abelhas que coletam néctar e pólen é às 10 horas;

A correlação entre a concentração de açúcares no néctar com a temperatura é direta, positiva e moderada e com a umidade relativa do ar é inversa, negativa e moderada.

\section{REFERÊNCIAS BIBLIOGRÁFICAS}

1. BOX, G. E. P.; HUNTER, W. G.; HUNTER, J. S. Statistics for experimenters, an introduction to design, data analysis, and model building. New York: John Wiley \& Sons, 1978. 537p.

2. CANE. J. H.; SCHIFFHAUER, S. Nectar production of cranberries: genotypic differences and insensitivity to soil fertility. Journal of American Society of Horticulture Science, Alexandria, v 122, n. 5, p. 665-667, 1997.

3. FUNARI, S. R. C.; BAUSB-VIANA, M. J.; CURI, P. R. FUNARI, A. R. M. Perfil diário de coleta de abelhas africanizadas (Apis mellifera L) na região de Botucatu SP. Bol. Ind. Anim., Nova Odessa, v. 53, n. 1, p. 99-103, 1996.

4. GOMES, M. F. F.;ALMEIDA, C. Q.; FAUSTINO, C. M. Levantamento das plantas apícola dos municípios de Nioaque e Rochedo no Estado de Mato Grosso do Sul. In: CONGRESSO BRASILEIRO DE APICULTURA, 12., 1998, Salvador. Anais... Salvador, CBA/ FAABA, 1998. p. 181.

5. JULIANO, J.C. Identificação de espécies de interesse apícola do Rio Grande do Sul In: CONGRESSO BRASILEIRO DE APICULTURA (2. : 1972 : Sete Lagoas). Anais. Sete Lagoas: G. W. Cosenza, 1972. p. 85-118.

6. LORENZI, H. Plantas daninhas do Brasil. Nova Odessa: Harri Lonenzi, 1982. 425p.

7. NICOLSON, S. W. Eucalyptus nectar: production, availability, composition and osmotic consequences for the larva of the eucalypt nectar fly, Drosophila flavohita Suid Afrikaanse. Tydskrif vir Wetenskap. Pretoria. v. 90, p. 75-79, 1994

8. NICOLSON, S.W. The importance of osmosis in nectar secretion and its consumption by insects. Am. Zool., Lawrence, v. 38, n. 9. p. 75-79, 1998.

9. PEGORARO, A.; CARPANEZZI, A. A. Avaliação do potencial melífero da bracatinga Revista do Setor de Ciências Agrárias, Curitiba. v. 14, n. 2, p. 167-172, 1995.

10. PEGORARO, A. Estudo da integração de diversos fatores no manejo de abelhas africanizadas Apis mellifera Linnaeus., 1758 (Hymenoptera: Apidae) na unidade fitogeográfica da floresta com araucária, no sul do Brasil. Curitiba, 2003 (Doutorado em Agronomia), Universidade Federal do Paraná. 148p.

11. WIESE, H. Nova apicultura. Guíba: Livraria e Editora Agropecuária Ltda, 1985. 292p.

Recebido em 05/03/2004 Aceito em 02/09/2005 